\title{
Nonperturbative features of the axial current
}

\author{
B. Z. Kopeliovich, Iván Schmidt and M. Siddikov \\ June 12, 2018 \\ Departamento de Física, Universidad Técnica Federico Santa María, \\ y Instituto de Estudios Avanzados en Ciencias e Ingeniería, \\ y Centro Científico - Tecnológico de Valparaíso, \\ Casilla 110-V, Valparaíso, Chile
}

\begin{abstract}
We study the nonperturbative structure of the axial current and evaluate the two-point light-cone distribution amplitudes (DA) associated with the correlator $\int d \xi e^{-i q \cdot \xi}\left\langle 0\left|\bar{\psi}(x) \Gamma \psi(y) J_{\mu}^{5}(\xi)\right| 0\right\rangle$ within the instanton vacuum model in the leading order in $\mathcal{O}\left(N_{c}\right)$. Due to the built-in chiral symmetry, four of eight axial DAs are related to that for pions. Knowledge of these nonperturbative objects is important for phenomenological study of high-energy neutrino interactions and semileptonic decays of heavy quarks. We provide a code for evaluation of these DAs and an interpolation formula valid in the region $Q^{2} \lesssim 1 \mathrm{GeV}^{2}$.
\end{abstract}

\section{Introduction}

According to the Standard Model, the weak interaction of leptons with quarks has a $V$ - $A$ structure. While the hadronic properties of the vector current has been well established in electro-magnetic processes, the structure of the axial current is less known, especially in the soft regime of low $Q^{2}$. At high energies and large $Q^{2}$ (small Bjorken $x$ ) one can rely on the well-known perturbative QCD expressions for the light-cone distribution functions (DA) derived in [1, 2, 3]. The difference between the DAs for the vector and axial currents reveals in different signs in front of the quark masses and some helicity components. However, in certain processes (e.g. neutrino-induced interactions, semileptonic decays of heavy bosons), which proceed at a low scale $Q^{2} \lesssim 1 \mathrm{GeV}^{2}$, the structures of the axial and vector currents are essentially different due to the spontaneous breaking of the chiral symmetry. At small $Q^{2} \lesssim m_{\pi}^{2}$ the axial current is dominated by the contribution of the pion pole. Presence of pions also affects the region of moderate $Q^{2}$ : axial-vector meson dominance does not work because of the $\rho \pi$ cut [4, 5, 6], which gives a larger contribution to the dispersion relation than the $a_{1}$ pole. 
A popular tool to describe the longitudinal component of the axial current is the phenomenological PCAC relation, which has been proposed in the pre-QCD era [7, 8, 9, 10]. It relates the longitudinal part of the interaction amplitude of the axial current with that of the pion [11. However, PCAC requires some model assumptions for continuation of the result to nonzero $Q^{2} \sim 1 \mathrm{GeV}^{2}$ and does not provide any information about the transverse part of the axial current.

In this paper we evaluate the two-point quark distribution amplitudes (DAs) of the axial current in the framework of the instanton vacuum model (see [12, 13, 14, and references therein). The important advantage of this model is the built-in dynamically broken chiral symmetry, which allows to reproduce the low-energy chiral structure of QCD. Recently this model was used for evaluation of the hadronic structure of the vector current [15, 16, pion DAs [17, 18, 19, 20, 21, 22], correlators of vector and axial currents [15, 23, 24], as well as different low-energy constants. The nonperturbative structure of the axial current is particularly important for processes which include soft kinematics or large distance between quarks. As was mentioned above, currently the axial distributions amplitudes can be accessed in the neutrino-hadron interactions (both inclusive [1, 2] and exclusive [34]), semileptonic decays of heavy quarks and other processes with production of charged dileptons $(l \nu)$ with small invariant mass $M_{l \nu}^{2} \approx 0$. Although there is a number of other processes which get contributions from the axial DAs (e.g. charged current mediated electroproduction), such processes are suppressed at small- $Q^{2}$ as $\sim Q^{4} / m_{W}^{4}$ compared to photonmediated analogs, and for large $Q^{2} \sim m_{W}^{2}$ all the nonperturbative effects in the wave functions become negligible.

The paper is organized as follows. In Section 2 we briefly overview the basic elements of the instanton vacuum model (IVM) used for further evaluations. In Section 3 we present definitions for the DAs. In Section 3.1 we discuss the constraints on the axial DAs imposed by the chiral symmetry and PCAC relation within the IVM model. The leading twist distribution amplitudes $\Phi_{\|}$and $\Phi_{\perp}$, which are the main result of the present paper, are derived in Section 3.2. In Section 3.3 we discuss the subleading twist DAs. We summarize the observations and make conclusions in Section 4.

\section{Instanton vacuum model}

The central object of the model is the effective action for the light quarks in the instanton vacuum, which in the leading order in $N_{c}$ has the form [13, 14, 25]

$$
S-S_{P T}=\int d^{4} x\left(\frac{N}{V} \ln \lambda+2 \Phi^{2}(x)+\bar{\psi}\left(\hat{p}+\hat{v}+\hat{a} \gamma_{5}-m-c \bar{L} f \otimes \Phi \cdot \Gamma_{m} \otimes f L\right) \psi\right)
$$

where $\Gamma_{m}$ is one of the matrices, $\Gamma_{m}=1, i \vec{\tau}, \gamma_{5}$, or $i \vec{\tau} \gamma_{5} ; \psi$ and $\Phi$ are the fields of constituent quarks and mesons respectively; $N / V$ is the density of the instanton gas; $\hat{v} \equiv v_{\mu} \gamma^{\mu}$ is the external vector current corresponding to the photon; $L$ is the gauge factor,

$$
\begin{aligned}
L(x, z) & =P \exp \left(i \int_{z}^{x} d \zeta^{\mu}\left(v_{\mu}(\zeta)+a_{\mu}(\zeta) \gamma_{5}\right)\right) \\
\bar{L}(x, z) & =\gamma_{0} L(x, z)^{\dagger} \gamma_{0}
\end{aligned}
$$


which provides the gauge covariance of the action [26, 27]. A formal derivation of the effective action (10), which starts from a minimal substitution in the QCD lagrangian and reproduces the effective action with gauge factors under standard assumptions like the zero-mode approximation for the single-instanton propagator, diluteness of the instanton vacuum and using the algorithm outlined in [13, 28, may be found in [25]. As was discussed in [28, violation of the gauge symmetry (in the absence of the gauge factors) originates from the zero mode approximation and is parametrically small, $\sim \rho^{2} / R^{2} \sim 0.1$. An alternative approach which does not rely on the zero-mode approximation was discussed in [29]. However, it includes direct resummation of the instanton propagators and demands very complicated calculations, even for the simplest correlators. A direct comparison of the two approaches is not trivial because in [29] a formal expansion parameter is $\mathcal{O}\left(\sqrt{N / V N_{c}}\right)$, not $\mathcal{O}\left(1 / N_{c}\right)$ as in [28]; however in the leading order numerically both approaches end up with close results [29].

Since in this paper we perform calculations in the leading-order in $\mathcal{O}\left(1 / N_{c}\right)$, for the sake of simplicity we do not include in (1) the terms with tensor couplings, which correspond to subleading non-planar diagrams, and neglected the finite width of the instanton size distribution. The function $f(p)$ in (1) is the Fourier transform of the zero-mode profile in the single-instanton background,

$$
f(p)=2 z\left(I_{0}(z) K_{1}(z)-I_{1}(z) K_{0}(z)-\frac{1}{z} I_{1}(z) K_{1}(z)\right)_{z=p \bar{\rho} / 2},
$$

with the high-energy asymptotic behaviour

$$
f(p) \sim \frac{6}{(p \bar{\rho})^{3}} .
$$

For the sake of simplicity, sometimes the dipole 13] and Gaussian [30] parameterizations of the formfactor are used,

$$
f(p)=\left\{\begin{array}{lc}
L^{2} /\left(L^{2}-p^{2}\right) & (\text { dipole }) \\
\exp \left(-p^{2} / L^{2}\right) & (\text { Gaussian })
\end{array}\right.
$$

with $L=\sqrt{2} / \bar{\rho} \sim 850 \mathrm{MeV}$. In Euclidean space, both functions are close to (44) and differ only at asymptotically high momenta. For most of the vacuum condensates, all three parametrizations (44 5) give close numbers. However, the distribution amplitudes, which are nonlocal quark operators with light-cone separation, are much more sensitive to the choice of the formfactor compared to the local vacuum condensates. Varying the formfactor $f(p)$ completely different results were obtained for the leading-twist pion DA in [18, 20, 31]. This happens because in the Taylor expansion of a nonlocal operator the higher moments are very sensitive to the endpoint behaviour of DAs, which, as will be discussed below, is controlled by the large-momentum asymptotics of the formfactor. In a special limiting case $f(p)=1$, which corresponds to the Nambu-Jona-Lasinio (NJL) model [32, 33], it is possible to eliminate the gauge factors $L, \bar{L}$ by gauge rotation, so this case corresponds to the local interaction of mesons with quarks.

In the leading order in $N_{c}$, we have the same Feynman rules as in perturbative theory, but with a momentum-dependent quark mass $\mu(p)$ in the quark propagator

$$
S(p)=\frac{1}{\hat{p}-\mu(p)+i 0} .
$$


The running mass of the constituent quark has a form

$$
\mu(p)=m+M f^{2}(p),
$$

where $m \approx 5 \mathrm{MeV}$ is the current quark mass, $M \approx 350 \mathrm{MeV}$ is the dynamical mass generated by the interaction with the instanton vacuum background. Due to presence of instantons the vector current quark coupling is also modified,

$$
\begin{aligned}
& \hat{v} \equiv v_{\mu} \gamma^{\mu} \Rightarrow \hat{V}=\hat{v}+\hat{V}^{n o n l} \\
& \hat{a} \equiv a_{\mu} \gamma^{\mu} \Rightarrow \hat{A}=\hat{a}+\hat{A}^{n o n l}
\end{aligned}
$$

In addition to the vertices present in perturbative QCD, the model has nonlocal terms with higher-order couplings of currents and mesons. The exact expressions for the nonlocal terms $\hat{V}^{\text {nonl }}, \hat{A}^{\text {nonl }}$ depend on the choice of the path in (2), and one can find in the literature different results [16, 20, 15, 25]. As was discussed in [25], this ambiguity arises due to the zero mode approximation employed in the derivation of (11). Since in the absence of the gauge links the violation of the chiral symmetry is parametrically suppressed as $\sim \rho^{2} / R^{2}$, the ambiguity in the choice of the path will affect the results only within the same limits. The longitudinal axial DAs are insensitive to this choice of the path at all. In what follows we employ the parameterizations,

$$
\begin{aligned}
& \hat{V}_{n o n l}=v_{\mu}\left(i M \frac{p_{1}^{\mu}+p_{2}^{\mu}}{p_{1}^{2}-p_{2}^{2}}\left(f\left(p_{1}\right)^{2}-f\left(p_{2}\right)^{2}\right)\right), \\
& \hat{A}_{n o n l}=a_{\mu}\left(i M \frac{p_{1}^{\mu}+p_{2}^{\mu}}{p_{1}^{2}-p_{2}^{2}}\left(f\left(p_{1}\right)-f\left(p_{2}\right)\right)^{2}\right)
\end{aligned}
$$

where $p_{1}, p_{2}$ are the momenta of the initial and final quarks.

\section{Distribution amplitudes for the axial current}

The DAs of the axial current are defined via 3-point correlators,

$$
\Psi_{\mu} \sim \int d^{4} \xi e^{-i q \cdot \xi}\left\langle 0\left|\bar{\psi}(y) \Gamma \psi(x) J_{\mu}^{5}(\xi)\right| 0\right\rangle
$$

where $x$ and $y$ are light-cone coordinates of the quark and antiquark, $q$ is the momentum flowing through the axial current and $\Gamma$ is one of the Dirac matrices, as was defined in (1). The structure of the axial current is different from the vector one because of the spontaneous chiral symmetry breaking and existence of near-massless pions. In particular, the axial current can fluctuate into a pion prior to the production of a $\bar{q} q$ pair. Therefore, the correlator (12) consists of two terms, schematically presented in the Figure 1 ,

One term comes from the combined contribution of the intermediate heavy states $\left(a_{1}\right.$ meson, $3 \pi$, etc.), and the other one represents fluctuations of the axial current into a pion. The chiral symmetry embedded 

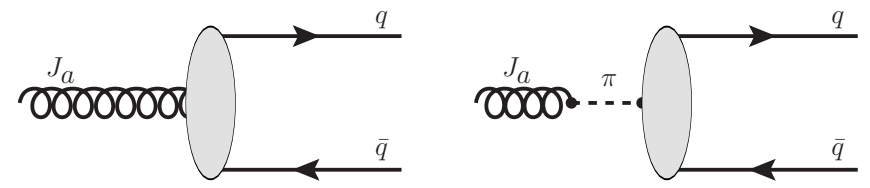

Figure 1: The DA contains two terms corresponding to either intermediate heavy axial states (left), or to a pion (right), which we label by bulk or pion respectively.

into the model relates the two terms as 1 ,

$$
\Psi_{\mu}=\Psi_{\mu}^{(b u l k)}+\Psi_{\mu}^{(\text {pion })}=\left(g_{\mu \nu}-\frac{q_{\mu} q_{\nu}}{q^{2}-m_{\pi}^{2}}\right) \Psi_{\nu}^{(b u l k)}
$$

This form of the DA explicitly satisfies PCAC. In what follows we concentrate on the part of the amplitude presented in the dispersion relation for the amplitude by the bulk of heavy states excluding the pion pole (left pane of Figure 10 [34, 35], tacitly assuming that the full DAs are given by Eq. (13). We define these DAs as 2 ,

$$
\begin{aligned}
& \int d^{4} x e^{-i q x}\left\langle 0\left|\bar{\psi}\left(-\frac{z}{2}\right)\left[-\frac{z}{2}, \frac{z}{2}\right] \gamma_{\mu} \gamma_{5} \psi\left(\frac{z}{2}\right) J_{\beta}^{5}(x)\right| 0\right\rangle=i f_{A} \int_{0}^{1} d \alpha e^{i(0.5-\alpha) p \cdot z} \times \\
& \times\left(\frac{p_{\mu} z_{\beta}}{p \cdot z} \Phi_{\|}(\alpha)+g_{\mu \beta}^{\perp} g_{\perp}^{(a)}(\alpha)\right. \\
& \left.+\frac{z_{\mu} z_{\beta}}{(p \cdot z)^{2}} g_{3}(\alpha)\right) \\
& \int d^{4} x e^{-i q x}\left\langle 0\left|\bar{\psi}\left(-\frac{z}{2}\right)\left[-\frac{z}{2}, \frac{z}{2}\right] \gamma_{\mu} \psi\left(\frac{z}{2}\right) J_{\beta}^{5}(x)\right| 0\right\rangle=-i f_{A} \epsilon_{\mu \beta \rho \sigma} p_{\rho} n_{\sigma} \int_{0}^{1} d \alpha e^{i(0.5-\alpha) p \cdot z} \frac{g_{\perp}^{(v)}(\alpha)}{4} \\
& \int d^{4} x e^{-i q x}\left\langle 0\left|\bar{\psi}\left(-\frac{z}{2}\right)\left[-\frac{z}{2}, \frac{z}{2}\right] \sigma_{\mu \nu} \gamma_{5} \psi\left(\frac{z}{2}\right) J_{\beta}^{5}(x)\right| 0\right\rangle=f_{A} \int_{0}^{1} d \alpha e^{i(0.5-\alpha) p \cdot z} \\
& \times \quad\left(\left(g_{\beta \mu}^{\perp} p_{\nu}-g_{\beta \nu}^{\perp} p_{\mu}\right) \Phi_{\perp}(\alpha)\right. \\
& +\frac{z_{\beta}}{(p \cdot z)^{2}}\left(p_{\mu} z_{\nu}-p_{\nu} z_{\mu}\right) h_{\|}^{(t)}(\alpha) \\
& \left.+\frac{1}{2}\left(g_{\beta \mu}^{\perp} z_{\nu}-g_{\beta \nu}^{\perp} z_{\mu}\right) \frac{1}{p \cdot z} h_{3}(\alpha)\right),
\end{aligned}
$$

\footnotetext{
${ }^{1} \mathrm{~A}$ formal proof of this statement is the same as the proof of transversity of the axial correlator $\left\langle a_{\mu} a_{\nu}\right\rangle$ in 25 ,

${ }^{2}$ Sometimes in the literature (see e.g. [36]) DAs $g_{\perp}^{(v)}, h_{\|}^{(p)}$ are defined with prefactor $\epsilon_{\mu \beta \rho \sigma} p_{\rho} z_{\sigma}$ in (15) and $z_{\beta}$ in (17). This redefinition corresponds to the change of the DA with its derivative. We've chosen definitions (15]17) in order to have relations between functions and not their derivatives in the PCAC relations (39,42).
} 


$$
\int d^{4} x e^{-i q x}\left\langle 0\left|\bar{\psi}\left(-\frac{z}{2}\right)\left[-\frac{z}{2}, \frac{z}{2}\right] \gamma_{5} \psi\left(\frac{z}{2}\right) J_{\beta}^{5}(x)\right| 0\right\rangle=f_{A} n_{\beta} \int_{0}^{1} d \alpha e^{i(0.5-\alpha) p \cdot z} \frac{h_{\|}^{(p)}(\alpha)}{2}
$$

where $q$ is the 4 -momentum carried by the axial current; $\alpha$ and $\bar{\alpha} \equiv 1-\alpha$ are the fractional light-cone momenta carried by the quark and antiquark; $z$ is the light-cone separation of the quark-antiquark, $z^{2}=0$; $p_{\mu}$ is the "positive direction" vector on the light-cone, $n_{\mu}$ is the "negative direction" vector on the light-cone, with normalization conditions $p \cdot n=1, p^{2}=n^{2}=0$. Transverse dimensions are chosen in such a way that the vector $q$ has only components in the $p, n$ - plane. Without any loss of generality, in what follows we choose a system where $q_{+}=q \cdot n=1$. All the DAs (14) contain a color gauge link

$$
\left[-\frac{z}{2}, \frac{z}{2}\right] \equiv P \exp \left(i \int_{-z / 2}^{z / 2} d \zeta_{\mu} A_{\mu}(\zeta)\right)
$$

In the instanton vacuum the gluon field $A_{\mu}(\zeta)$ is a sum of the fields of individual instantons and antiinstantons. As was discussed in [37], the contribution of this link to the twist-two operators is parametrically suppressed as $(\rho / R)^{4}$ and numerically is negligible. However for higher twists this gauge link contribution might be important. In general, such calculation is quite complicated and is doable only in the so-called single-instanton approximation [14]. In what follows, for the sake of simplicity we drop the gauge links. For this reason, the higher-twist DAs should be considered only as an order of magnitude estimates. The normalization constant $f_{A}$ is a dimensional parameter introduced in order to make the distribution amplitudes dimensionless. Its value is fixed by the condition

$$
\int_{0}^{1} d \alpha \Phi_{\|}\left(\alpha, Q^{2}=0\right)=1
$$

If we define an "effective" axial meson state vector $\left|A^{(\lambda)}(q)\right\rangle$ as

$$
\left|A^{(\lambda)}(q)\right\rangle=\int d^{4} x e^{-i q \cdot x} e_{\beta}^{(\lambda)}(q) J_{\beta}^{5}(x)|0\rangle,
$$

where the polarization vectors $e^{(\lambda)}$ are defined as 38

$$
\begin{aligned}
& e_{\mu}^{(\lambda=\|)}=e^{(\lambda)} \cdot n\left(p_{\mu}-\frac{q^{2}}{2} n_{\mu}\right), \\
& p \cdot e_{\mu}^{(\lambda=\perp)}=n \cdot e_{\mu}^{(\lambda=\perp)}=0, \\
& \left|e^{(\perp)}(q)\right|^{2}=-1,
\end{aligned}
$$

then we may rewrite Eqns. (23, 26) in a standard form as distribution amplitudes of the effective axial meson state [36], 


$$
\begin{aligned}
\left\langle 0\left|\bar{\psi}(y) \gamma_{\mu} \gamma_{5} \psi(x)\right| A(q)\right\rangle & =i f_{A} \int_{0}^{1} d \alpha e^{i(0.5-\alpha) p \cdot z} \times \\
& \times\left(p_{\mu} \frac{e^{(\lambda)} \cdot z}{p \cdot z} \Phi_{\|}(\alpha)+e_{\mu}^{(\lambda=\perp)} g_{\perp}^{(a)}(\alpha)+z_{\mu} \frac{e^{(\lambda)} \cdot z}{(p \cdot z)^{2}} g_{3}(\alpha)\right), \\
\left\langle 0\left|\bar{\psi}(y) \gamma_{\mu} \psi(x)\right| A(q)\right\rangle & =-i f_{A} \epsilon_{\mu \nu \rho \sigma} e_{\nu}^{(\lambda)} p_{\rho} z_{\sigma} \int_{0}^{1} d \alpha e^{i(0.5-\alpha) p \cdot z} \frac{g_{\perp}^{(v)}(\alpha)}{4} \\
\left\langle 0\left|\bar{\psi}(y) \sigma_{\mu \nu} \gamma_{5} \psi(x)\right| A(q)\right\rangle= & f_{A} \int_{0}^{1} d \alpha e^{i(0.5-\alpha) p \cdot z}\left(\left(e_{\mu}^{(\lambda=\perp)} p_{\nu}-e_{\nu}^{(\lambda=\perp)} p_{\mu}\right) \Phi_{\perp}(\alpha)+\right. \\
+ & \frac{e^{(\lambda)} \cdot z}{(p \cdot z)^{2}}\left(p_{\mu} z_{\nu}-p_{\nu} z_{\mu}\right) h_{\|}^{(t)}(\alpha) \\
& \left.+\frac{1}{2}\left(e_{\mu}^{(\lambda)} z_{\nu}-e_{\nu}^{(\lambda)} z_{\mu}\right) \frac{1}{p \cdot z} h_{3}(\alpha)\right), \\
\left\langle 0\left|\bar{\psi}(y) \gamma_{5} \psi(x)\right|\right. & A(q)\rangle=f_{A} e^{(\lambda)} \cdot n \int_{0}^{1} d \alpha e^{i(0.5-\alpha) p \cdot z} \frac{h_{\|}^{(p)}(\alpha)}{2} .
\end{aligned}
$$

The distribution amplitudes $\Phi_{\|}(\alpha), \Phi_{\perp}(\alpha)$ are of twist-2; $g_{\perp}^{(a)}, g_{\perp}^{(v)}, h_{\|}^{(t)}, h_{\|}^{(p)}$ are of twist-3; $g_{3}, h_{3}$ are of twist-4. Chiral parity: all wave functions in (23), (24) are chiral even, all wave functions in (25), (26) are chiral odd.

Taking the limit $z \rightarrow 0$ in (14,17) and using the low-energy expansions dictated for the correlators by chiral symmetry [39]

$$
\begin{aligned}
i \int d^{4} x e^{i q x}\left\langle 0\left|J_{\mu}^{5, a}(x) J_{\nu}^{5, b}(0)\right| 0\right\rangle & =\delta^{a b} f_{\pi}^{2}\left(g_{\mu \nu}-\frac{q_{\mu} q_{\nu}}{q^{2}-m_{\pi}^{2}}\right)+\mathcal{O}\left(q^{2}, m_{\pi}^{2}\right), \\
i \int d^{4} x e^{i q x}\left\langle 0\left|J_{\mu}^{5, a}(x) J^{5, b}(0)\right| 0\right\rangle & =-i \delta^{a b} f_{\pi} G_{\pi} \frac{q_{\mu}}{q^{2}-m_{\pi}^{2}}+\mathcal{O}\left(q^{2}, m_{\pi}^{2}\right), \\
G_{\pi} \delta^{a b} & \equiv\left\langle 0\left|J^{5, a}\right| \pi^{b}(q)\right\rangle=\underbrace{\frac{f_{\pi} m_{\pi}^{2}}{m}}_{2 B f_{\pi}} \delta^{a b}+\mathcal{O}\left(q^{2}, m_{\pi}^{2}\right),
\end{aligned}
$$

we may obtain the following normalization conditions for the distribution amplitudes at $Q^{2}=0$ :

$$
\int d \alpha \Phi_{\|}(\alpha)=1, \quad \int d \alpha g_{a}^{(\perp)}(\alpha)=1, \quad \int d \alpha g_{3}(\alpha)=0, \quad \int d \alpha h_{\|}^{(p)}(\alpha)=1 .
$$


As was discussed in [36], similar to the vector channel, for axial case approximate Wandzura-Wilczek type relations,

$$
\begin{aligned}
& g_{\perp}^{(a), W W}(\alpha) \approx \frac{1}{2}\left[\int_{0}^{u} \frac{d v}{\bar{v}} \Phi_{\|}(v)+\int_{u}^{1} \frac{d v}{v} \Phi_{\|}(v)\right], \\
& g_{\perp}^{(v), W W}(\alpha) \approx 2\left[-\int_{0}^{u} \frac{d v}{\bar{v}} \Phi_{\|}(v)+\int_{u}^{1} \frac{d v}{v} \Phi_{\|}(v)\right], \\
& h_{\|}^{(t), W W}(\alpha) \approx(2 \alpha-1)\left[-\int_{0}^{u} \frac{d v}{\bar{v}} \Phi_{\perp}(v)+\int_{u}^{1} \frac{d v}{v} \Phi_{\perp}(v)\right], \\
& h_{\|}^{(p), W W}(\alpha) \approx 2\left[\int_{0}^{u} \frac{d v}{\bar{v}} \Phi_{\perp}(v)-\int_{u}^{1} \frac{d v}{v} \Phi_{\perp}(v)\right],
\end{aligned}
$$

can be valid under assumptions that twist-three quark-gluon DAs are zero.

Let us consider briefly the endpoint behaviour of the DAs ( $\alpha$ or $\bar{\alpha} \sim \rho^{2} / R^{2} \sim 0.1$ ). In general case, in the leading order over $1 / N_{c}$, the DAs have a structure

$$
\Phi\left(\alpha, q^{2}\right) \sim \int \frac{d l^{-} d^{2} l_{\perp}}{(2 \pi)^{3}} \frac{\mathcal{F}(l, q)}{\left(l^{2}+\mu^{2}(l)\right)\left((l+q)^{2}+\mu^{2}(l+q)\right)}-\left.\frac{\mathcal{G}(l, q)}{\left(l^{2}+m^{2}\right)\left((l+q)^{2}+m^{2}\right)}\right|_{l^{+}=-\alpha q^{+}},
$$

where the functions $\mathcal{F}(l, q), \mathcal{G}(l, q)$ depend on the DA in question, and we assume that

$$
\begin{aligned}
\mathcal{F}(l, q) & =\mathcal{F}_{0}\left(l^{2},(l+q)^{2}, q^{2}\right)+\mathcal{F}_{1}\left(l^{2},(l+q)^{2}, q^{2}\right) l^{-}, \\
\mathcal{G}(l, q) & =\mathcal{G}_{0}\left(l^{2},(l+q)^{2}, q^{2}\right)+\mathcal{G}_{1}\left(l^{2},(l+q)^{2}, q^{2}\right) l^{-} .
\end{aligned}
$$

In the limit $f(p) \rightarrow 0$ the running quark mass $\mu(p) \rightarrow m$, and $\mathcal{F} \equiv \mathcal{G}$. First we consider the case when the function $\mathcal{G}(l, q)$ is zero, at least near the endpoints. In order to analyze the endpoint behaviour of the first term in (32), we follow [18] and for simplicity take $\mu(l) \approx \mu(l+q) \approx M=$ const in the denominator. In this case integration over $l^{-}$is rather straightforward and yields

$$
\begin{aligned}
\Phi\left(\alpha, q^{2}\right) & \sim \frac{1}{2 q^{+}} \int \frac{d^{2} l_{\perp}}{(2 \pi)^{2}}\left(\left.\frac{\mathcal{F}_{0}-\frac{l_{\perp}^{2}+M^{2}}{2 \alpha} \mathcal{F}_{1}}{\alpha \bar{\alpha} q^{2}-l_{\perp}^{2}-M^{2}}\right|_{l^{+}=-\alpha q^{+}, l^{-}=-\frac{l_{\perp}^{2}+M^{2}}{2 \alpha q^{+}}}\right. \\
& \left.-\left.\frac{\mathcal{G}_{0}-\frac{l_{\perp}^{2}+m^{2}}{2 \alpha} \mathcal{G}_{1}}{\alpha \bar{\alpha} q^{2}-l_{\perp}^{2}-m^{2}}\right|_{l^{+}=-\alpha q^{+}, l^{-}=-\frac{l_{\perp}^{2}+m^{2}}{2 \alpha q^{+}}}\right), \\
\left.l^{2}\right|_{l^{+}=-\alpha q^{+}, l^{-}=-\frac{l_{\perp}^{2}+M^{2}}{2 \alpha q^{+}}} & =M^{2}, \\
\left.(l+q)^{2}\right|_{l^{+}=-\alpha q^{+}, l^{-}=-\frac{l_{\perp}^{2}+M^{2}}{2 \alpha q^{+}}} & =\bar{\alpha} q^{2}-\frac{\bar{\alpha}}{\alpha}\left(l_{\perp}^{2}+M^{2}\right) .
\end{aligned}
$$


As one can see, the first term in (33) has a branch cut which starts at $q^{2}=4 M^{2}$. This is due to the fact that in the instanton vacuum model there is no built-in confinement. In what follows we assume that $q^{2}<4 M^{2}$ and will use (33) in order to discuss the endpoint behaviour of the corresponding DAs in the following sections. As we will see, this behaviour is extremely sensitive to the parametrization of the formfactor $f(p)$. In pQCD limit $\mathcal{F}(l, q) \sim 1$ or $\sim l_{\perp}^{2}$, so the integral diverges. For the DAs discussed in this paper $\mathcal{F}_{0}(l, q)$ has a form

$$
\mathcal{F}_{0} \sim f^{m}(l) f^{n}(l+q), \quad m, n \geq 0
$$

so the corresponding contribution to $\Phi(l, q)$ in the small- $\alpha$ region is

$$
\Phi(\alpha, q) \sim\left\{\begin{array}{cc}
\alpha^{n}, & \text { (Dipole) } \\
\alpha^{3 n / 2}, & (\text { Parametrization(4) }) \\
\exp \left(\frac{\text { const }}{\alpha}\right), & \text { (Gaussian) }
\end{array}\right.
$$

The function $\mathcal{F}_{1}(l, q)$ is a constant (does not depend on $l$ ) in most of the integrals discussed below, $\mathcal{F}_{1}(l, q)=\mathcal{F}_{1}$, so taking into account $\mathcal{F}_{1}=\mathcal{G}_{1}$, we see that the contribution $\sim \mathcal{F}_{1}$ is finite,

$$
\Phi(\alpha, q) \sim q^{2} \mathcal{F}_{1} \ln \left(\frac{M^{2}-q^{2} \alpha \bar{\alpha}}{m^{2}-q^{2} \alpha \bar{\alpha}}\right)
$$

The second term in (33) may be integrated directly, yielding in the infrared region 3

$$
\sim \ln \left(m^{2}-\alpha \bar{\alpha} q^{2}\right)
$$

which is logarithmically divergent in the chiral limit and exceeds the finite endpoint contribution given by (36) for $\alpha<m^{2} / q^{2}$.

\subsection{On PCAC relation}

The PCAC hypothesis was proposed in the pre-QCD era [7, 8, 9, 10] and has been intensively used as a phenomenological tool for describing the longitudinal part of the axial hadronic current at small virtualities. In operator form, the PCAC relation is

$$
\partial_{\mu} J_{\mu}^{5, a}=f_{\pi} m_{\pi}^{2} \phi^{a}
$$

where $J_{\mu}^{5, a}$ is the axial current, $\phi^{a}$ is the effective pion field, and $f_{\pi}$ is the pion decay constant. The PCAC relation (38) allows to relate four of eight the axial DAs, Eqs. (47), (52), (53) and (56), with corresponding pion DAs,

$$
\begin{aligned}
f_{A} \Phi_{\|}\left(\alpha, q^{2}=m_{\pi}^{2}\right) & =f_{\pi}^{2} \sqrt{2} \phi_{2 ; \pi}(\alpha) \\
f_{A} g_{3}\left(\alpha, q^{2}=m_{\pi}^{2}\right) & =\frac{f_{\pi}^{2} \sqrt{2}}{2} \psi_{4 ; \pi}(\alpha)
\end{aligned}
$$

\footnotetext{
${ }^{3}$ The coefficient in front of the $\log$ in (37) depends on the exact structure of $\mathcal{G}_{0,1}$ and will be discussed below
} 


$$
\begin{aligned}
& f_{A} h_{\|}^{(t)}\left(\alpha, q^{2}=m_{\pi}^{2}\right)=-\frac{\sqrt{2}}{3} \frac{f_{\pi}^{2} m_{\pi}^{2}}{m_{u}+m_{d}} \phi_{3 ; \pi}^{(\sigma)}(\alpha) \\
& f_{A} h_{\|}^{(p)}\left(\alpha, q^{2}=m_{\pi}^{2}\right)=\frac{2 \sqrt{2} f_{\pi}^{2} m_{\pi}^{2}}{m_{u}+m_{d}} \phi_{3 ; \pi}^{(p)}(\alpha)
\end{aligned}
$$

where the pion DAs are defined as [40, 34,

$$
\begin{aligned}
\left\langle 0\left|\bar{\psi}(y) \gamma_{\mu} \gamma_{5} \psi(x)\right| \pi(q)\right\rangle & =i f_{\pi} \sqrt{2} \int_{0}^{1} d \alpha e^{i(\alpha p \cdot y+\bar{\alpha} p \cdot x)} \times \\
& \times\left(p_{\mu} \phi_{2 ; \pi}(\alpha)+\frac{1}{2} \frac{z_{\mu}}{(p \cdot z)} \psi_{4 ; \pi}(\alpha)\right), \\
\left\langle 0\left|\bar{\psi}(y) \gamma_{5} \psi(x)\right| \pi(q)\right\rangle= & -i f_{\pi} \sqrt{2} \frac{m_{\pi}^{2}}{m_{u}+m_{d}} \int_{0}^{1} d \alpha e^{i(\alpha p \cdot y+\bar{\alpha} p \cdot x)} \phi_{3 ; \pi}^{(p)}(\alpha), \\
\left\langle 0\left|\bar{\psi}(y) \sigma_{\mu \nu} \gamma_{5} \psi(x)\right| \pi(q)\right\rangle= & -\frac{i}{3} f_{\pi} \sqrt{2} \frac{m_{\pi}^{2}}{m_{u}+m_{d}} \int_{0}^{1} d \alpha e^{i(\alpha p \cdot y+\bar{\alpha} p \cdot x)} \times \\
\times & \frac{1}{p \cdot z}\left(p_{\mu} z_{\nu}-p_{\nu} z_{\mu}\right) \phi_{3 ; \pi}^{(\sigma)}(\alpha) .
\end{aligned}
$$

It worth to mention that the relations (39,42) may be obtained using instead of (38) model-independent $\partial_{\mu} J_{\mu}^{5, a}(x)=2 i m J^{5, a}(x)$, where $J^{5, a}(x)$ is pseudoscalar-isovector current, and taking afterwards a residue at the pion pole. The DAs of the pion Eqs. (43)-(45) were discussed in detail in [15, 16, 20]. The leading order expressions for these DAs in the framework of the IVM are presented in Appendix A.

Since both $\Phi_{\|}\left(\alpha, q^{2}=m_{\pi}^{2}\right) \approx \Phi_{\|}(\alpha, 0)$ and $\phi_{2 ; \pi}(\alpha)$ are normalized to unity, we immediately conclude that

$$
f_{A}=\sqrt{2} f_{\pi}^{2}
$$

which is confirmed numerically.

In the framework of the instanton vacuum model, the relations (39,42) are satisfied exactly due to the built-in chiral symmetry and transverse structure of the corresponding DAs (13). However, in the following sections, we will consider separately the "transverse" part $\Psi_{\nu}^{(b u l k)}$ and the corresponding pion DA. The purpose of this exercise is to demonstrate that the DAs indeed have the transverse structure (13)).

\subsection{Leading-twist distribution amplitudes}

In the leading twist there are two independent distribution amplitudes, symmetric $\Phi_{\|}(\alpha)$ and antisymmetric $\Phi_{\perp}(\alpha)$. Straightforward evaluation yields for $\Phi_{\|}$

$$
\Phi_{\|}\left(\alpha, q^{2}\right)=\frac{1}{i f_{A}} \int d^{4} x e^{-i q x} \int \frac{d z}{2 \pi} e^{i(\alpha-0.5) p \cdot z}\left\langle 0\left|\bar{\psi}\left(-\frac{z}{2}\right) \gamma_{+} \gamma_{5} \psi\left(\frac{z}{2}\right) J_{-}^{5}(x)\right| 0\right\rangle
$$




$$
\begin{aligned}
& =\frac{8 N_{c}}{f_{A}} \int \frac{d l^{-} d^{2} l_{\perp}}{(2 \pi)^{4}}\left[\frac{\mu(l) \mu(l+q)+l_{\perp}^{2}+\alpha \bar{\alpha} q^{2}}{\left(l^{2}+\mu^{2}(l)\right)\left((l+q)^{2}+\mu^{2}(l+q)\right)}-\frac{m^{2}+l_{\perp}^{2}+\alpha \bar{\alpha} q^{2}}{\left(l^{2}+m^{2}\right)\left((l+q)^{2}+m^{2}\right)}\right. \\
& \left.-\frac{M(f(l+q)-f(l))^{2}\left(2 l^{-}+q^{2} \alpha\right)(\mu(l) \bar{\alpha}+\mu(l+q) \alpha)}{\left((l+q)^{2}-l^{2}\right)\left(l^{2}+\mu^{2}(l)\right)\left((l+q)^{2}+\mu^{2}(l+q)\right)}\right]_{l^{+}=-\alpha q^{+}}
\end{aligned}
$$

where the constant $f_{A}$ is fixed from the normalization condition (18),

$$
f_{A}=8 N_{c} \int \frac{d^{4} l}{(2 \pi)^{4}}\left[\frac{\mu^{2}(l)+l_{\perp}^{2}}{\left(l^{2}+\mu^{2}(l)\right)^{2}}-\frac{M\left(f^{\prime}(l)\right)^{2} l^{-}}{l^{2}\left(l^{2}+\mu^{2}(l)\right)^{2}}\right]
$$

As was discussed in the Section 3.1, due to PCAC relation $f_{A}=\sqrt{2} f_{\pi}^{2}$, which is confirmed numerically.

In Figure 2 the DA $\Phi_{\|}(\alpha)$ is plotted as function of $\alpha$ for several values of $Q^{2}=-q^{2}$. Near the endpoints, this distribution amplitude vanishes for all three parametrizations (415). Indeed, using the algorithm discussed at the beginning of this section, the DA may be approximated with (33), where the function $\mathcal{F}(l, q)$ after some simplifications can be reduced to

$$
\mathcal{F}(l, q) \sim f^{2}(l) f(l+q)[f(l+q)-f(l)],
$$

which yields the near-endpoint asymptotics $\Phi_{\|}(\alpha) \sim(\alpha \bar{\alpha})^{3 / 2}$ for the parametrization (44), $\Phi_{\|}(\alpha) \sim \alpha \bar{\alpha}$ for the dipole parametrization, and non-analytic $\Phi_{\|}(\alpha) \sim \exp \left(M^{2} / L^{2} \alpha\right)$ for the Gaussian parametrization. As was discussed in Section 3.1, this DA is equal to the pion DA $\phi_{2 ; \pi}(\alpha)$. In the right pane of the Figure 2 we see that this is indeed the case (small deviations are due to the finite precision of numerical calculations).

The antisymmetric DA $\Phi_{\perp}(\alpha)$ has a form

$$
\begin{aligned}
\Phi_{\perp}\left(\alpha, q^{2}\right) & =\frac{g_{\beta \mu} n_{\nu}-g_{\beta \nu} n_{\mu}}{4 f_{A}} \int d^{4} x e^{-i q x} \int \frac{d z}{2 \pi} e^{i(\alpha-0.5) p \cdot z}\left\langle 0\left|\bar{\psi}\left(-\frac{z}{2} n\right) \sigma_{\mu \nu} \gamma_{5} \psi\left(\frac{z}{2} n\right) J_{\beta}^{5}(x)\right| 0\right\rangle \\
& =\frac{8 N_{c}}{f_{A}} \int \frac{d l_{-} d^{2} l_{\perp}}{(2 \pi)^{3}}\left[\frac{-\alpha \mu(l+q)+\bar{\alpha} \mu(l)}{\left(l^{2}+\mu^{2}(l)\right)\left((l+q)^{2}+\mu^{2}(l+q)\right)}\right. \\
& \left.+\frac{l_{\mu_{\perp}}^{2}}{(l+q)^{2}-l^{2}} \frac{M(f(l+q)-f(l))^{2}}{\left(l^{2}+\mu^{2}(l)\right)\left((l+q)^{2}+\mu^{2}(l+q)\right)}\right]_{l^{+}=-\alpha q^{+}}
\end{aligned}
$$

In Figure 3 the distribution amplitude is shown for several values of $Q^{2}$. Notice that according to Eq. (49), at the endpoints the function does not depend on $q^{2}$ and has a finite limit.

In Appendix B we provide interpolation formulas for fast estimates of the DAs $\Phi_{\|}, \Phi_{\perp}$. 

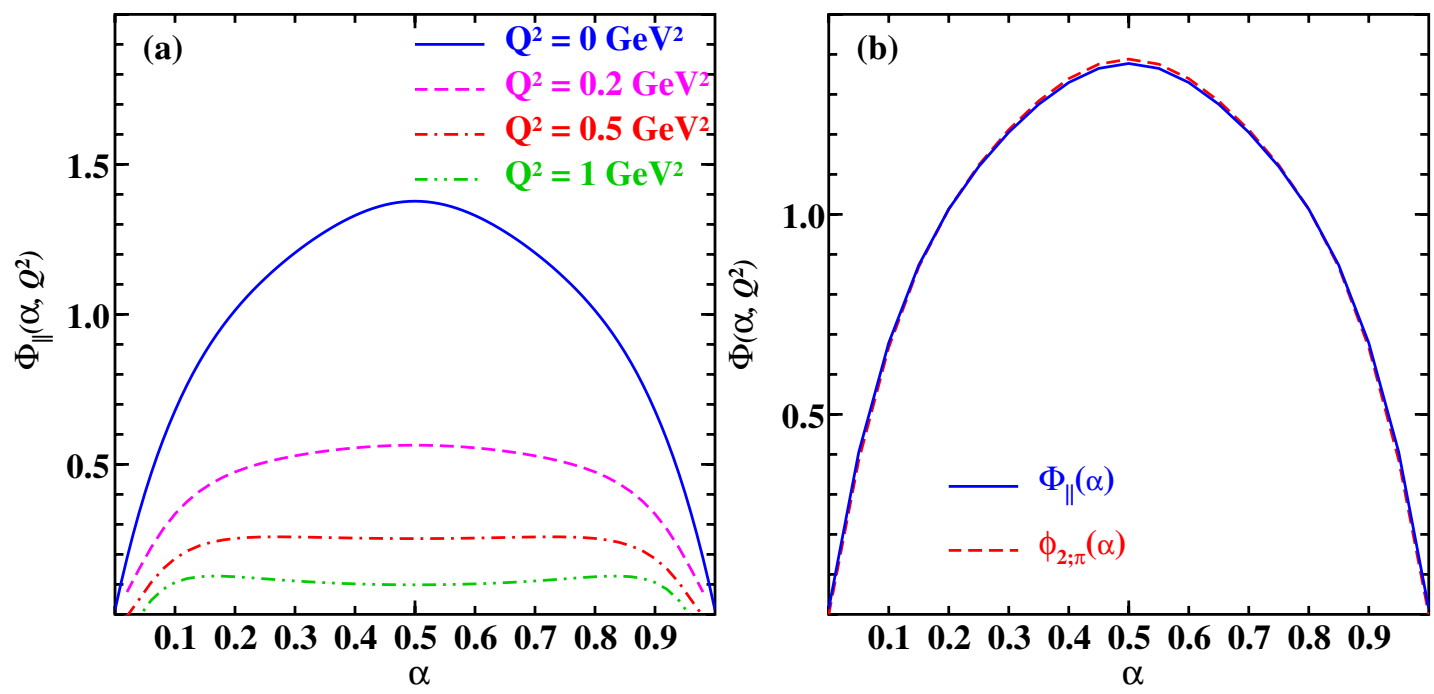

Figure 2: (color online) (a) Dependence of the distribution amplitude $\Phi_{\|}$on $\alpha$ for several values of $Q^{2}$. (b) Comparison of the DA $\Phi_{\|}$with pion DA $\phi_{2 ; \pi}$. Small deviation from PCAC relation (39) is due to finite numerical precision of the result.

\subsection{Higher twist distribution amplitudes}

In twist-three there are four independent distribution amplitudes $g_{\perp}^{(a)}, g_{\perp}^{(v)}, h_{\|}^{(t)}, h_{\|}^{(p)}$. Straightforward evaluation of the twist-3 DAs yields

$$
\begin{aligned}
g_{\perp}^{(a)}\left(\alpha, q^{2}\right) & =\frac{8 N_{c}}{i f_{A}} \int \frac{d l^{-} d^{2} l_{\perp}}{(2 \pi)^{4}}\left[\frac{\mu(l) \mu(l+q)+(2 \alpha-1) l_{-} q_{+}+\alpha \frac{q^{2}}{2}}{\left(l^{2}+\mu^{2}(l)\right)\left((l+q)^{2}+\mu^{2}(l+q)\right)}-\frac{m^{2}+(2 \alpha-1) l_{-} q_{+}+\alpha \frac{q^{2}}{2}}{\left(l^{2}+m^{2}\right)\left((l+q)^{2}+m^{2}\right)}(50)\right. \\
& \left.+\frac{l_{\mu_{\perp}}^{2}}{(l+q)^{2}-l^{2}} \frac{M(f(l+q)-f(l))^{2}(\mu(l+q)-\mu(l))}{\left(l^{2}+\mu^{2}(l)\right)\left((l+q)^{2}+\mu^{2}(l+q)\right)}\right]_{l^{+}=-\alpha q^{+}} \cdot \\
g_{\perp}^{(v)}\left(\alpha, q^{2}\right)= & -\frac{2 \epsilon_{\mu \beta \rho \sigma} p_{\rho} n_{\sigma}}{i f_{A}} \int d^{4} x e^{-i q x} \int \frac{d z}{2 \pi} e^{i(\alpha-0.5) p \cdot z}\left\langle 0\left|\bar{\psi}\left(-\frac{z}{2}\right) \gamma_{\mu} \psi\left(\frac{z}{2}\right) J_{\beta}^{5}(x)\right| 0\right\rangle \\
= & \frac{16 N_{c}}{f_{A}} \int \frac{d l^{-} d^{2} l_{\perp}}{(2 \pi)^{4}}\left[\frac{q^{2}}{\left(l^{2}+l_{-} q_{+}(l)\right)\left((l+q)^{2}+\mu^{2}(l+q)\right)}-\frac{q^{2}}{\left(l^{2}+m^{2}\right)\left((l+q)^{2}+m^{2}\right)}\right]_{l^{+}=-\alpha q^{+}} \\
h_{\|}^{(t)}\left(\alpha, q^{2}\right) & =\frac{\left(p_{\nu} n_{\rho}-p_{\rho} n_{\nu}\right) p_{\beta}}{2 f_{A}} \int d^{4} x e^{-i q x} \int \frac{d z}{2 \pi} e^{i(\alpha-0.5) p \cdot z}\left\langle 0\left|\bar{\psi}\left(-\frac{z}{2} n\right) \sigma_{\nu \rho} \gamma_{5} \psi\left(\frac{z}{2} n\right) J_{\beta}^{5}(x)\right| 0\right\rangle
\end{aligned}
$$




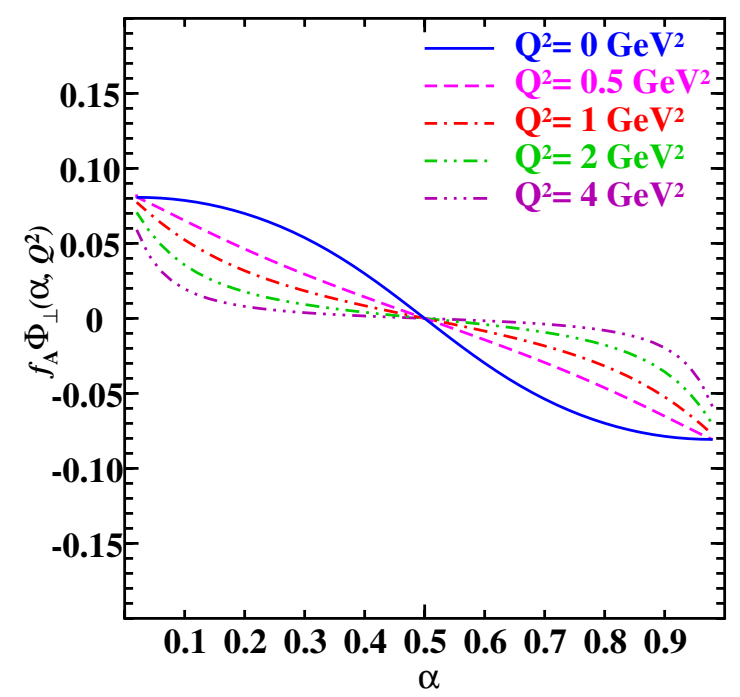

Figure 3: (color online) Dependence of the distribution amplitude $\Phi_{\perp}$ on $\alpha$ for several values of $Q^{2}$.

$$
\begin{aligned}
& =-\frac{8 N_{c}}{f_{A}} \int \frac{d l^{-} d^{2} l_{\perp}}{(2 \pi)^{4}}\left[\frac{\mu(l+q) l_{-}+\mu(l)\left(l^{-}+\frac{q^{2}}{2}\right)}{\left(l^{2}+\mu^{2}(l)\right)\left((l+q)^{2}+\mu^{2}(l+q)\right)}-\frac{m\left(2 l^{-}+\frac{q^{2}}{2}\right)}{\left(l^{2}+m^{2}\right)\left((l+q)^{2}+m^{2}\right)}(52)\right. \\
& \left.+\frac{2 M\left(l^{-}+\frac{q^{2}}{4}\right)\left(l^{-}+\alpha \frac{q^{2}}{2}\right)(f(l+q)-f(l))^{2}}{\left((l+q)^{2}-l^{2}\right)\left(l^{2}+\mu^{2}(l)\right)\left((l+q)^{2}+\mu^{2}(l+q)\right)}\right]_{l^{+}=-\alpha q^{+}} \\
& h_{\|}^{(p)}\left(\alpha, q^{2}\right)=-\frac{2 p_{\beta}}{f_{A}} \int d^{4} x e^{-i q x} \int \frac{d z}{2 \pi} e^{i(\alpha-0.5) p \cdot z}\left\langle 0\left|\bar{\psi}\left(-\frac{z}{2}\right) \gamma_{5} \psi\left(\frac{z}{2}\right) J_{\beta}^{5}(x)\right| 0\right\rangle \\
& =-\frac{16 N_{c}}{f_{A}} \int \frac{d l^{-} d^{2} l_{\perp}}{(2 \pi)^{4}}\left[\frac{(\mu(l)-\mu(l+q))\left(l^{-}+\alpha \frac{q^{2}}{2}\right)}{\left(l^{2}+\mu^{2}(l)\right)\left((l+q)^{2}+\mu^{2}(l+q)\right)}\right. \\
& \left.+\frac{\left(l_{\perp}^{2}+(1-2 \alpha) l^{-}-\alpha \frac{q^{2}}{2}+\mu(l) \mu(l+q)\right)}{(l+q)^{2}-l^{2}} \frac{M(f(l+q)-f(l))^{2}\left(2 l^{-}+q^{2} \alpha\right)}{\left(l^{2}+\mu^{2}(l)\right)\left((l+q)^{2}+\mu^{2}(l+q)\right)}\right]_{l^{+}=-\alpha q^{+}}
\end{aligned}
$$

Using the algorithm discussed at the beginning of this section, we may get that the endpoint behaviour of these DA is controlled by the contribution of the type (37) which behaves for $q^{2} \neq 0$ as $\log \left(m^{2}+q^{2} \alpha \bar{\alpha}\right)$,

$$
g_{\perp}^{(a)}\left(\alpha, q^{2}\right) \sim\left[2 m^{2}+q^{2}\left(\alpha^{2}+\bar{\alpha}^{2}\right)\right] \ln \left(m^{2}+q^{2} \alpha \bar{\alpha}\right) .
$$




$$
\begin{gathered}
g_{\perp}^{(v)}\left(\alpha, q^{2}\right) \sim q^{2}(2 \alpha-1) \ln \left(m^{2}+q^{2} \alpha \bar{\alpha}\right) . \\
h_{\|}^{(t)}\left(\alpha, q^{2}\right) \sim(2 \alpha-1) \log \left(M^{2}+q^{2} \alpha \bar{\alpha}\right) .
\end{gathered}
$$

In the Figures 4, 5 the distribution amplitudes (50,53) are shown as a function of $\alpha$ for several values of $Q^{2}$. In the same Figure 4 one can see the prediction of the Wandzura-Wilczek approximation (28,31). As one can see, this approximation gives results inconsistent with prediction of the present approach. The reason is that the twist-3 quark-gluon operators $\bar{\psi}(x) \gamma_{\alpha} G_{\mu \nu}(x)[x, y] \psi(y)$ disregarded in the Wandzura-Wilczek approximation are not zero in the instanton vacuum. Similarly, the Wandzura-Wilczek approximation fails for the vector current DAs [16].

While evaluation of the twist-3 DAs was done consistently, for practical applications the DAs should be evolved to higher scales. In contrast to a simple ERBL evolution of the leading twist distribution amplitudes, higher-twist DAs mix with twist-3 three-parton quark-gluon operators. Although evaluation of the latter is possible in the instanton vacuum [14], it is considerably more involved and is beyond the scope of this paper.
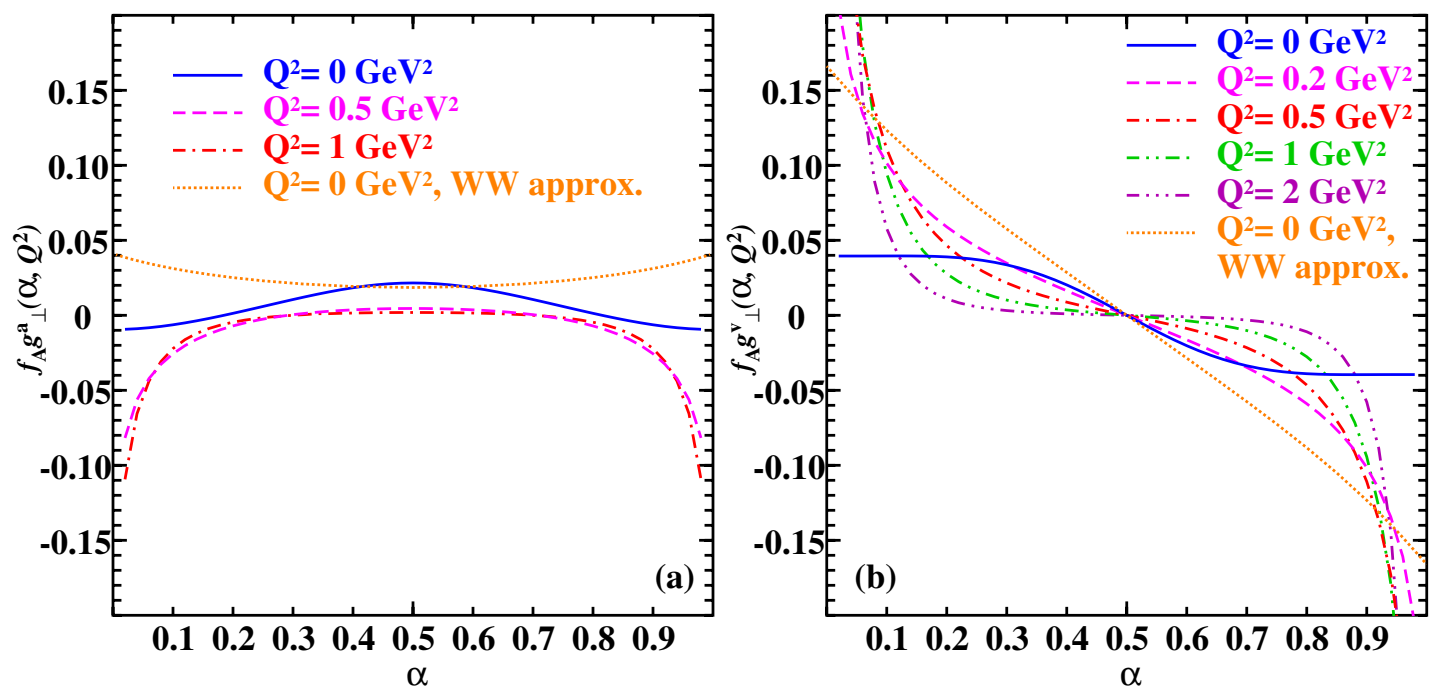

Figure 4: (color online) (a) Dependence of the distribution amplitude $g_{\perp}^{a}$ on $\alpha$ for several values of $Q^{2}$. (b) Dependence of the distribution amplitude $g_{\perp}^{v}$ on $\alpha$ for several values of $Q^{2}$. In both plots dotted line is a prediction of the Wandzura-Wilczek approximation (28]29).

Finally, for the sake of completeness, below we give the expressions for the twist-4 distribution amplitudes. Up to the best of our knowledge, there is no any model estimates for these functions. Also, as was discussed at the beginning of this section, for the twist-4 DAs omission of the gauge link may be not justified, so these DAs should be considered as an order-of-magnitude estimates. 

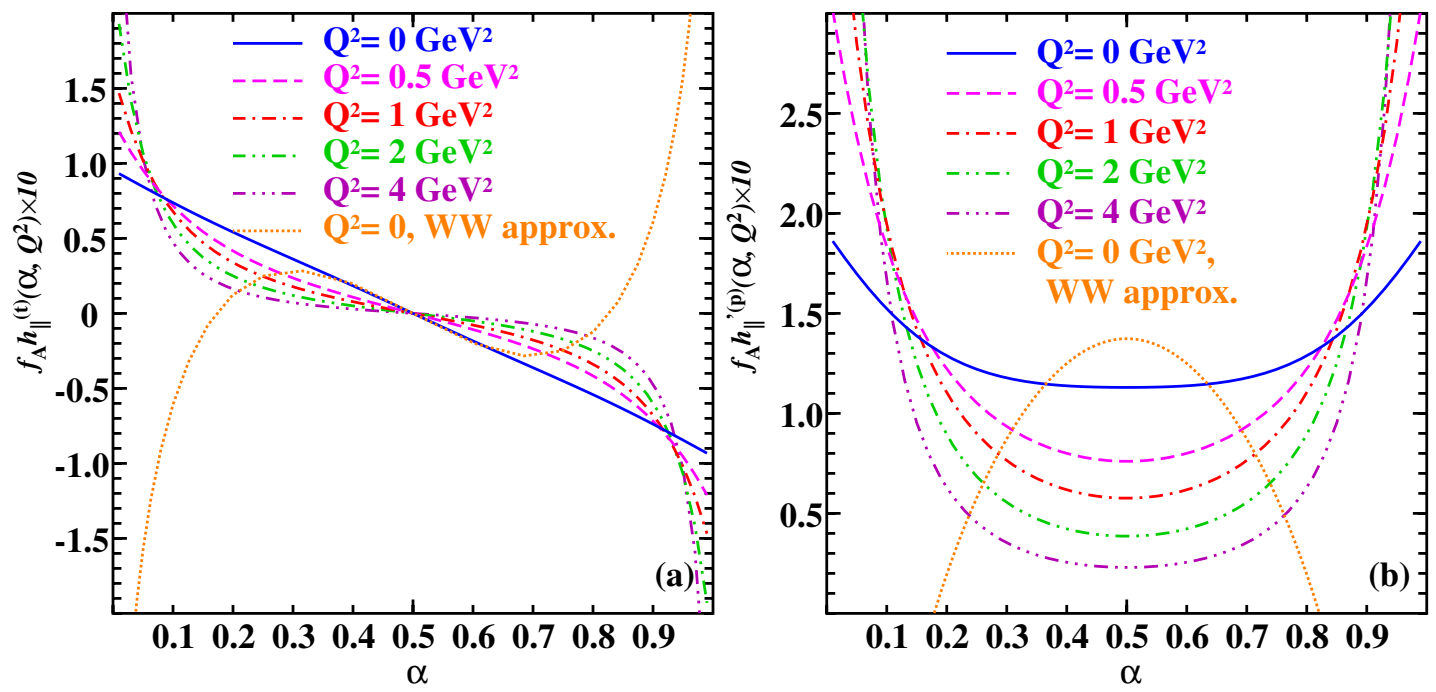

Figure 5: (color online) (a) Dependence of the distribution amplitude $h_{\|}^{(t)}$ on $\alpha$ for several values of $Q^{2}$. (b) Dependence of the distribution amplitude $h_{\|}^{(p)}$ on $\alpha$ for several values of $Q^{2}$. In both plots dotted line is a prediction of the Wandzura-Wilczek approximation (30131).

Straightforward evaluation yields for the DAs

$$
\begin{aligned}
g_{3}\left(\alpha, q^{2}\right) & =\int d^{4} x e^{-i q x} \int \frac{d z}{2 \pi} e^{i(\alpha-0.5) p \cdot z}\left\langle 0\left|\bar{\psi}\left(-\frac{z}{2}\right) \gamma_{-} \gamma_{5} \psi\left(\frac{z}{2} n\right) J_{-}^{5}(x)\right| 0\right\rangle \\
& =\frac{8 N_{c}}{f_{A}} \int \frac{d l^{-} d^{2} l_{\perp}}{(2 \pi)^{4}}\left[\frac{2\left(l^{-}\right)^{2}+l^{-} q^{2}}{\left(l^{2}+\mu^{2}(l)\right)\left((l+q)^{2}+\mu^{2}(l+q)\right)}-\frac{2\left(l^{-}\right)^{2}+l^{-} q^{2}}{\left(l^{2}+m^{2}\right)\left((l+q)^{2}+m^{2}\right)}\right. \\
& \left.+\frac{\left(\mu(l)\left(l^{-}+\frac{q^{2}}{2}\right)-\mu(l+q) l^{-}\right)}{\left((l+q)^{2}-l^{2}\right)} \frac{M(f(l+q)-f(l))^{2}\left(2 l^{-}+\frac{q^{2}}{2}\right)}{\left(l^{2}+\mu^{2}(l)\right)\left((l+q)^{2}+\mu^{2}(l+q)\right)}\right]_{l^{+}=-\alpha q^{+}} \\
h_{3}\left(\alpha, q^{2}\right) & =\frac{g_{\beta \mu} p_{\nu}-g_{\beta \nu} p_{\mu}}{4 f_{A}} \int d^{4} x e^{-i q x} \int \frac{d z}{2 \pi} e^{i(\alpha-0.5) p \cdot z}\left\langle 0\left|\bar{\psi}\left(-\frac{z}{2} n\right) \sigma_{\mu \nu} \gamma_{5} \psi\left(\frac{z}{2} n\right) J_{\beta}^{5}(x)\right| 0\right\rangle \\
& =\frac{8 N_{c}}{f_{A}} \int \frac{d l_{-} d^{2} l_{\perp}}{(2 \pi)^{3}}\left[\frac{l_{-} \mu(l+q)+\left(l_{-}+q_{-}\right) \mu(l)}{\left(l^{2}+\mu^{2}(l)\right)\left((l+q)^{2}+\mu^{2}(l+q)\right)}-\frac{\left(2 l_{-}+q_{-}\right) m}{\left(l^{2}+m^{2}\right)\left((l+q)^{2}+m^{2}\right)}\right.
\end{aligned}
$$




$$
\left.+\frac{1}{2} \frac{l_{\mu_{\perp}}^{2} q^{2}}{(l+q)^{2}-l^{2}} \frac{M(f(l+q)-f(l))^{2}}{\left(l^{2}+\mu^{2}(l)\right)\left((l+q)^{2}+\mu^{2}(l+q)\right)}\right]_{l^{+}=-\alpha q^{+}}
$$

In Figure 6 the distribution amplitudes (56/57) are shown for several values of $Q^{2}$. Near the endpoints, these functions are finite. Straightforward integration confirms the sum rule (27) for the $g_{3}$.

As was discussed in Section 3.1, the PCAC imposes relations between the longitudinal DAs $h_{\|}^{(p)}, h_{\|}^{(t)}, g_{3}$ and corresponding pion DAs. In the Figure 6 we show that these relations are indeed satisfied (small deviations are due to the finite precision of numerical calculations).
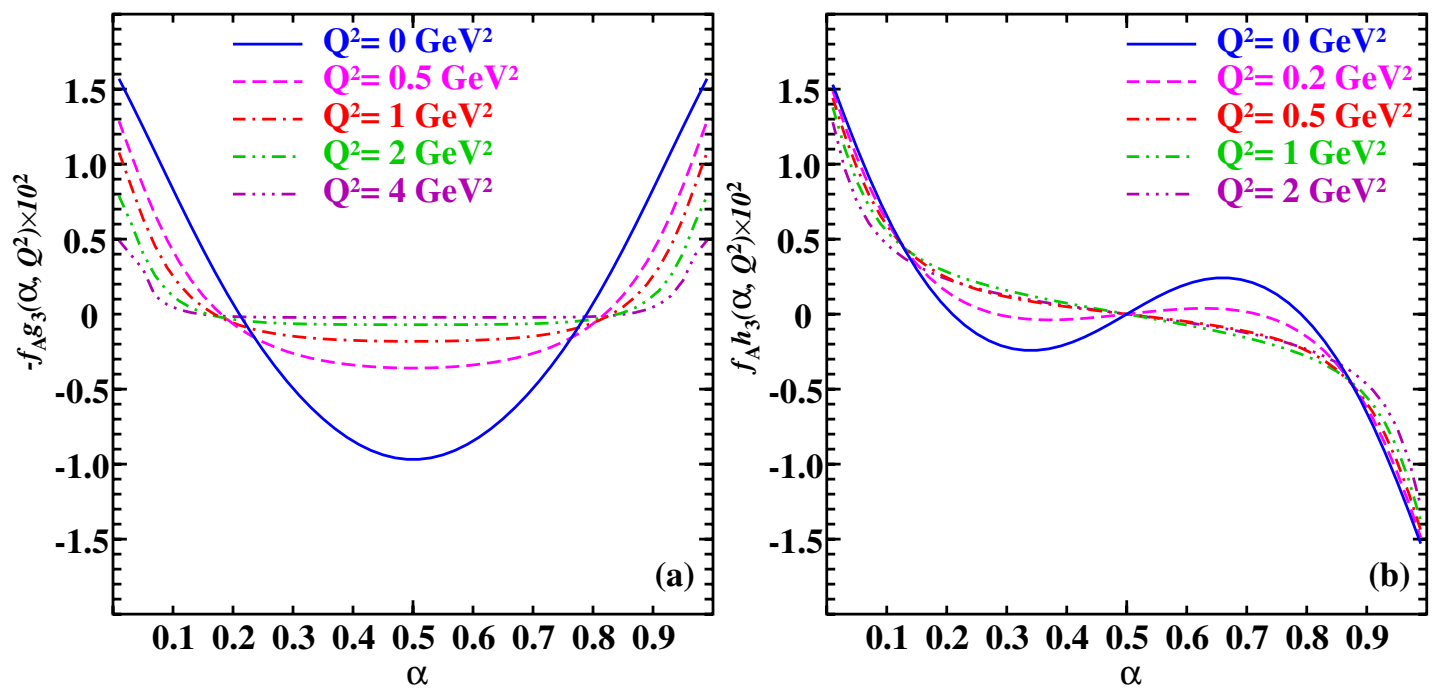

Figure 6: (color online) (a) Dependence of the distribution amplitude $g_{3}$ on $\alpha$ for several values of $Q^{2}$. (b) Dependence of the distribution amplitude $h_{3}$ on $\alpha$ for several values of $Q^{2}$.

\section{Summary}

We studied the properties of the axial current and evaluated the related DAs in the framework of the instanton vacuum model. These DAs are calculated at the low initial scale $\left(\sim \rho^{-1}=600 \mathrm{MeV}\right)$ and can be evolved up to a higher scale employing the standard tools. The built-in chiral symmetry leads to the PCAC-type relations between the DAs of the axial current and pion.

The main result of the paper, the leading twist DAs $\Phi_{\|}, \Phi_{\perp}$ were presented in Section 3.2. We found that the leading-twist DA $\Phi_{\|}(\alpha)$, which is sensitive to the choice of the formfactor, has a shape close to the asymptotic form, which vanishes at the endpoints for all possible formfactors. The transverse DA $\Phi_{\perp}(\alpha)$ is antisymmetric and is finite near the endpoints, as discussed in Section 3.2 For both DAs we provide simple interpolation parametrizations (62)69), which allow fast numerical evaluations. 

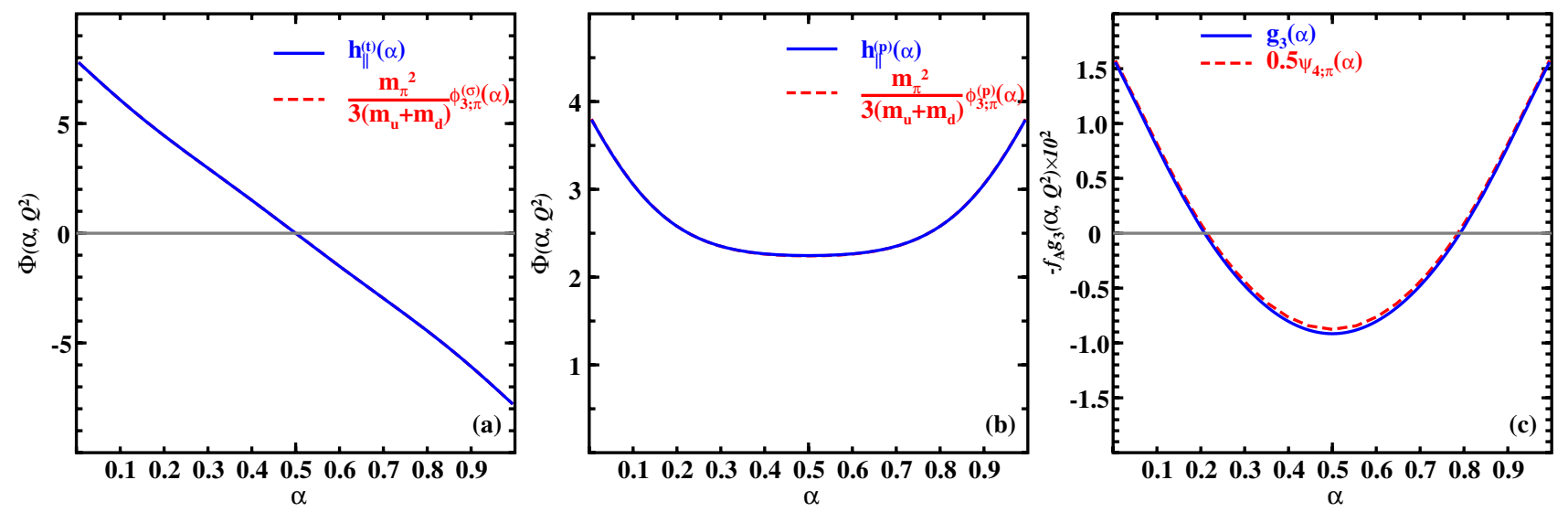

Figure 7: (color online) Comparison of the longitudinal DAs $h_{\|}^{(p)}, h_{\|}^{(t)}, g_{3}$ with corresponding pion DAs. As follows from the PCAC relations (40,42), the two curves almost coincide (deviations are less than one percent).

The subleading twist DAs (see Sections 3.3) discussed in Section 3.3, were compared with the predictions based on the popular Wandzura-Wilczek type relations (28) 31). We found that these relations are inconsistent with prediction of the present approach. The reason is that the twist-3 quark-gluon operators $\bar{\psi}(x) \gamma_{\alpha} G_{\mu \nu}(x)[x, y] \psi(y)$, disregarded in the Wandzura-Wilczek approximation, are sizable in the instanton vacuum. Similarly, the Wandzura-Wilczek approximation fails for the vector current DAs [16]. We check that the PCAC-type relations between longitudinal DAs of the axial current and of the pion are satisfied for all DAs.

For further practical applications we provide a computational code.

\section{Acknowledgements}

We are grateful to A. E. Dorokhov for the fruitful discussion of the distribution amplitudes. We acknowledge the partial support by Fondecyt (Chile) grants No. 1090291, 1100287 and 1120920.

\section{A Pion distribution amplitudes}

The distribution amplitudes of the pion (43)-(45) and their evaluation in the framework of the IVM was performed and discussed in detail in [17, 20, 22, 21, 15, 16]. For the sake of completeness we present here the leading-order expressions for the pion distribution amplitudes.

$$
\phi_{2 ; \pi}(\alpha)=\frac{1}{i f_{\pi} \sqrt{2}} \int \frac{d z}{2 \pi} e^{i(\alpha-0.5) p \cdot z}
$$




$$
\begin{aligned}
& \times\left\langle 0\left|\bar{\psi}\left(-\frac{z}{2} n\right) \hat{n} \gamma_{5} \psi\left(\frac{z}{2} n\right)\right| \pi(q)\right\rangle= \\
& =\frac{8 N_{c}}{f_{\pi} \sqrt{2}} \int \frac{d l^{-} d^{2} l_{\perp}}{(2 \pi)^{4}} \\
& \times\left[M f(l) f(l+q) \frac{\mu(l) \bar{\alpha}+\mu(l+q) \alpha}{\left(l^{2}+\mu^{2}(l)\right)\left((l+q)^{2}+\mu^{2}(l+q)\right)}\right]_{l^{+}=-\alpha q^{+}} . \\
& \psi_{4 ; \pi}(\alpha)=\frac{\sqrt{2}}{i f_{\pi}} \int \frac{d z}{2 \pi} e^{i(\alpha-0.5) p \cdot z} \\
& \times\left\langle 0\left|\bar{\psi}\left(-\frac{z}{2} n\right) \hat{p} \gamma_{5} \psi\left(\frac{z}{2} n\right)\right| \pi(q)\right\rangle \\
& =\frac{16 N_{c}}{f_{\pi} \sqrt{2}} \int \frac{d l^{-} d^{2} l_{\perp}}{(2 \pi)^{4}} \\
& \times\left[M f(l) f(l+q) \frac{\mu(l)\left(l_{-}+q_{-}\right)-\mu(l+q) l_{-}}{\left(l^{2}+\mu^{2}(l)\right)\left((l+q)^{2}+\mu^{2}(l+q)\right)}\right]_{l^{+}=-\alpha q^{+}} . \\
& \phi_{3 ; \pi}^{(p)}(\alpha)=\frac{1}{f_{\pi} \sqrt{2}} \frac{m_{u}+m_{d}}{m_{\pi}^{2}} \int \frac{d z}{2 \pi} e^{i(\alpha-0.5) p \cdot z} \\
& \times\left\langle 0\left|\bar{\psi}\left(-\frac{z}{2} n\right) \gamma_{5} \psi\left(\frac{z}{2} n\right)\right| \pi(q)\right\rangle \\
& =\frac{8 N_{c}}{f_{\pi} \sqrt{2}} \frac{m_{u}+m_{d}}{m_{\pi}^{2}} \int \frac{d l^{-} d^{2} l_{\perp}}{(2 \pi)^{4}} M f(l) f(l+q) \\
& \times\left[\frac{\mu(l) \mu(l+q)+l^{2}+l \cdot q}{\left(l^{2}+\mu^{2}(l)\right)\left((l+q)^{2}+\mu^{2}(l+q)\right)}\right]_{l^{+}=-\alpha q^{+}} . \\
& \phi_{3 ; \pi}^{(\sigma)}(\alpha)=\frac{3 i}{2 \sqrt{2} f_{\pi}} \frac{m_{u}+m_{d}}{m_{\pi}^{2}}\left(p_{\mu} n_{\nu}-p_{\nu} n_{\mu}\right) \int \frac{d z}{2 \pi} e^{i(\alpha-0.5) p \cdot z} \\
& \times\left\langle 0\left|\bar{\psi}\left(-\frac{z}{2} n\right) \sigma_{\mu \nu} \gamma_{5} \psi\left(\frac{z}{2} n\right)\right| \pi(q)\right\rangle \\
& =-\frac{24 N_{c}}{f_{\pi} \sqrt{2}} \frac{m_{u}+m_{d}}{m_{\pi}^{2}} \int \frac{d l^{-} d^{2} l_{\perp}}{(2 \pi)^{4}} M f(l) f(l+q) \\
& \times\left[\frac{q_{+} l_{-}+\frac{q^{2}}{2} \alpha}{\left(l^{2}+\mu^{2}(l)\right)\left((l+q)^{2}+\mu^{2}(l+q)\right)}\right]_{l^{+}=-\alpha q^{+}} .
\end{aligned}
$$




\section{B Interpolating expressions for the leading twist distribution ampli- tudes}

The distribution amplitudes $\Phi_{\|}, \Phi_{\perp}$ are given by Eqs. (47/49). For numerical calculations involving these DAs, it is convenient to have approximate interpolating expressions. In this section we construct such interpolation using expansion over the Gegenbauer polynomials.

For the DA $\Phi_{\|}$such expansion has a form

$$
\Phi_{\|}\left(\alpha, Q^{2}, \mu^{2} \approx \rho^{-2}\right)=6 x(1-x) \sum_{n=e v e n} a_{n}\left(\mu^{2} \approx \rho^{-2}, Q^{2}\right) C_{n}^{3 / 2}(2 x-1) .
$$

Since the DA $\Phi_{\|}$in the small- $Q^{2}$ region is close to the asymptotic form, we may approximate it taking the first few terms in a series (62). The coefficients $a_{n}$ in the region $Q^{2} \lesssim 1 \mathrm{GeV}^{2}$ may be approximated as

$$
\begin{aligned}
& a_{0}\left(\mu^{2} \approx \rho^{-2}, Q^{2}\right) \approx \frac{0.189}{Q^{2}+0.177}-0.068, \\
& a_{2}\left(\mu^{2} \approx \rho^{-2}, Q^{2}\right) \approx \frac{0.071}{Q^{2}+0.665}-0.034, \\
& a_{4}\left(\mu^{2} \approx \rho^{-2}, Q^{2}\right) \approx \frac{0.028}{Q^{2}+0.496}-0.039, \\
& a_{6}\left(\mu^{2} \approx \rho^{-2}, Q^{2}\right) \approx \frac{0.21}{Q^{2}+2.5}-0.081, \\
& a_{8}\left(\mu^{2} \approx \rho^{-2}, Q^{2}\right) \approx \frac{0.347}{Q^{2}+3.939}-0.083,
\end{aligned}
$$

where we have explicitly shown dependence on the intrinsic scale $\mu^{2} \sim \rho^{-2} \approx 0.6 \mathrm{GeV}^{2}$ to avoid confusion with virtuality $Q^{2}$. As we can see from (63)67), at small $Q^{2}$ the series coefficients decrease rapidly and the omitted terms give a negligible correction.

Similarly, the Gegenbauer expansion of the DA $\Phi_{\perp}\left(\alpha, q^{2}\right)$ has a form

$$
\Phi_{\perp}\left(\alpha, Q^{2}, \mu^{2} \approx \rho^{-2}\right)=6 x(1-x) \sum_{n=o d d} a_{n}\left(\mu^{2} \approx \rho^{-2}, Q^{2}\right) C_{n}^{3 / 2}(2 x-1),
$$

where due to to asymmetry ony odd polynomials contribute. However, since the DA $\Phi_{\perp}\left(\alpha, q^{2}\right)$ does not vanish at the endpoints, the Gegenbauer series (68) converges very slowly. In order to have a simple and practical approximation, we subtract the linear function $l(x)=c(2 x-1)$ and end up with an approximate parametrization

$$
\Phi_{\perp}\left(\alpha, Q^{2}, \mu^{2} \approx \rho^{-2}\right) \approx c(2 x-1)+6 x(1-x) \sum_{n=o d d} \tilde{a}_{n}\left(\mu^{2} \approx \rho^{-2}, Q^{2}\right) C_{n}^{3 / 2}(2 x-1)
$$

where

$$
\tilde{a}_{1}\left(\mu^{2} \approx \rho^{-2}, Q^{2}\right) \approx 2.195-\frac{5.645}{Q^{2}+2.069}
$$




$$
\begin{aligned}
\tilde{a}_{3}\left(\mu^{2} \approx \rho^{-2}, Q^{2}\right) & \approx \frac{0.703}{Q^{2}+0.585}-\frac{3.629}{\left(Q^{2}+1.765\right)^{2}}, \\
\tilde{a}_{5}\left(\mu^{2} \approx \rho^{-2}, Q^{2}\right) & \approx \frac{0.0056}{Q^{2}+0.216}-0.038 \\
c & \approx-3.17 .
\end{aligned}
$$

and the omitted terms give a negligible correction.

\section{References}

[1] R. Fiore and V. R. Zoller, Phys. Lett. B 632 (2006) 87 [hep-ph/0509097].

[2] R. Fiore and V. R. Zoller, JETP Lett. 82 (2005) 385 [Pisma Zh. Eksp. Teor. Fiz. 82 (2005) 440] hep-ph/0508187.

[3] L. Motyka and G. Watt, Phys. Rev. D 78 (2008) 014023 arXiv:0805.2113 [hep-ph]].

[4] R. T. Deck, Phys. Rev. Lett. 13 (1964) 169.

[5] B. Z. Kopeliovich and P. Marage, Int. J. Mod. Phys. A 8 (1993) 1513.

[6] B. Z. Kopeliovich, I. K. Potashnikova, I. Schmidt and J. Soffer, Phys. Rev. D 84 (2011) 114012 arXiv:1109.2500 [hep-ph].

[7] Y. Nambu, Phys. Rev. Lett. 4, 380 (1960).

[8] J. Goldstone, A. Salam and S. Weinberg, Phys. Rev. 127, 965 (1962).

[9] M. L. Goldberger and S. B. Treiman, Phys. Rev. 110, 1178 (1958).

[10] J. T. Goldman, M. Duong-van and R. Blankenbecler, Phys. Rev. D 20, 619 (1979).

[11] S. L. Adler, Phys. Rev. 135, B963 (1964).

[12] T. Schäfer and E. V. Shuryak, Rev. Mod. Phys. 70 (1998) 323 [arXiv:hep-ph/9610451].

[13] D. Diakonov and V. Y. Petrov, Nucl. Phys. B 272 (1986) 457

[14] D. Diakonov, M. V. Polyakov and C. Weiss, Nucl. Phys. B 461 (1996) 539 arXiv:hep-ph/9510232.

[15] A. E. Dorokhov and W. Broniowski, Eur. Phys. J. C 32 (2003) 79 arXiv:hep-ph/0305037.

[16] A. E. Dorokhov, W. Broniowski and E. Ruiz Arriola, Phys. Rev. D 74 (2006) 054023 arXiv:hep-ph/0607171.

[17] S. V. Esaibegian and S. N. Tamarian, Sov. J. Nucl. Phys. 51 (1990) 310 [Yad. Fiz. 51 (1990) 485]. 
[18] V. Y. Petrov, M. V. Polyakov, R. Ruskov, C. Weiss and K. Goeke, Phys. Rev. D 59 (1999) 114018 arXiv:hep-ph/9807229].

[19] I. V. Anikin, A. E. Dorokhov, L. Tomio, Phys. Lett. B475 (2000) 361. hep-ph/9909368.

[20] I. V. Anikin, A. E. Dorokhov and L. Tomio, Phys. Part. Nucl. 31 (2000) 509 [Fiz. Elem. Chast. Atom. Yadra 31 (2000) 1023].

[21] A. E. Dorokhov, JETP Lett. 77 (2003) 63 [Pisma Zh. Eksp. Teor. Fiz. 77 (2003) 68] arXiv:hep-ph/0212156.

[22] A. E. Dorokhov and L. Tomio, Phys. Rev. D 62 (2000) 014016.

[23] A. E. Dorokhov, Eur. Phys. J. C 42 (2005) 309 arXiv:hep-ph/0505007.

[24] A. E. Dorokhov, Int. J. Mod. Phys. A 20 (2005) 631 arXiv:hep-ph/0408003.

[25] K. Goeke, M. M. Musakhanov and M. Siddikov, Phys. Rev. D 76 (2007) 076007 arXiv:0707.1997 [hep-ph]]

[26] J. Terning, Phys. Rev. D 44 (1991) 887.

[27] R. D. Bowler and M. C. Birse, Nucl. Phys. A 582 (1995) 655 arXiv:hep-ph/9407336.

[28] D. Diakonov and V. Y. Petrov, Sov. Phys. JETP 62 (1985) 431 [Zh. Eksp. Teor. Fiz. 89 (1985) 751].

[29] P. V. Pobylitsa, Phys. Lett. B 226 (1989) 387.

[30] B. Golli, W. Broniowski and G. Ripka, Phys. Lett. B 437 (1998) 24 arXiv:hep-ph/9807261.

[31] A. E. Dorokhov, Czech. J. Phys. 56 (2006) $\quad$ F169 [Braz. J. Phys. 37 (2007) 819] arXiv:hep-ph/0610212.

[32] Y. Nambu and G. Jona-Lasinio, Phys. Rev. 122, 345 (1961).

[33] Y. Nambu and G. Jona-Lasinio, Phys. Rev. 124, 246 (1961).

[34] B. Z. Kopeliovich, I. Schmidt and M. Siddikov, Phys.Rev. D 84 (2011) 033012 arXiv:1107.2845 [hep-ph]].

[35] B. Z. Kopeliovich, I. K. Potashnikova, I. Schmidt and M. Siddikov, Phys. Rev. C 84, 024608 (2011) arXiv:1105.1711 [hep-ph]].

[36] K. C. Yang, Nucl. Phys. B 776 (2007) 187 arXiv:0705.0692 [hep-ph]].

[37] M. V. Polyakov and C. Weiss, Acta Phys. Polon. B 28 (1997) 2751 arXiv:hep-ph/9709436.

[38] P. Ball, V. M. Braun, Y. Koike and K. Tanaka, Nucl. Phys. B 529 (1998) 323 arXiv:hep-ph/9802299. 
[39] J. Gasser, H. Leutwyler, Annals Phys. 158 (1984) 142

[40] P. Ball, V. M. Braun and A. Lenz, JHEP 0605 (2006) 004 arXiv:hep-ph/0603063. 